\title{
Inter-Operator Variability in Perfusion Assessment of Tumors in MRI Using Automated AIF Detection
}

\author{
Edward Ashton ${ }^{1}$, Teresa McShane ${ }^{2}$, and Jeffrey Evelhoch ${ }^{3}$ \\ ${ }^{1}$ VirtualScopics, LLC, 350 Linden Oaks, Rochester, NY, 14580, USA \\ ashton@virtualscopics.com \\ ${ }^{2}$ Pfizer Global Research and Development, Eastern Point Road, Groton, CT, 06340, USA \\ teresa.m.mcshane@pfizer.com \\ ${ }^{3}$ Amgen, One Amgen Center Drive, Thousand Oaks, CA, 91320, USA \\ evelhocheamgen.com
}

\begin{abstract}
A method is presented for the calculation of perfusion parameters in dynamic contrast enhanced MRI. This method requires identification of enhancement curves for both tumor tissue and plasma. Inter-operator variability in the derived rate constant between plasma and extra-cellular extra-vascular space is assessed in both canine and human subjects using semi-automated tumor margin identification with both manual and automated arterial input function (AIF) identification. Experimental results show a median coefficient of variability (CV) for parameter measurement with manual AIF identification of $21.5 \%$ in canines and $11 \%$ in humans, with a median $\mathrm{CV}$ for parameter measurement with automated AIF identification of $6.7 \%$ in canines and $6 \%$ in humans.
\end{abstract}

\section{Introduction}

Dynamic contrast enhanced MRI (DCE-MRI) has demonstrated considerable utility in both diagnosing and evaluating the progression and response to treatment of malignant tumors.[1,2] DCE-MRI involves the periodic acquisition of $\mathrm{T}_{1}$-weighted images before, during and after injection of a gadolinium labeled tracer such as gadopentetate dimeglumine. The change over time in signal intensity in a voxel or region of interest in this time series can then be related to tracer concentration. By making use of a two-compartment model, with one compartment representing blood plasma and the other extra-vascular extra-cellular space (EES), the observed enhancement curves in tissue and plasma can be used to estimate various physiological parameters $[3,4,5,6]$.

The parameter of primary interest in this work is the volume transfer constant between blood plasma and EES, commonly referred to as $K^{\text {trans }}$ [7]. This parameter is related to both blood flow and endothelial permeability-surface area product, and is therefore a good endpoint for assessing the blood supply to a target malignancy.

One of the primary challenges in estimating perfusion parameters is identifying an accurate arterial input function (AIF). One common approach is to avoid this problem by making use of a general concentration-time curve such as that utilized by Weinmann et al. [8], as described by Tofts and Kermonde [9]. However, using a 
theoretical AIF ignores differences in injection rate and cardiac output, which may vary from subject to subject and even with a single subject over time [10]. These differences can greatly reduce measurement reproducibility. Galbraith et al. have reported thresholds for statistically significant change over time in $K^{\text {trans }}$ in a single tumor using this method of $-45 \%$ and $+83 \%$ [11]. A second option is for an analyst to draw a manual ROI within an artery, and use the mean enhancement curve within that ROI as the subject-specific AIF, as described by Vonken et al. [12]. This approach is complicated by the fact that the MR signal in arteries is frequently corrupted by flow artifacts, with the result that regions of interest at different points in the same artery or in other nearby vessels can provide grossly different enhancement curves. It should be noted that these artifacts are sequence dependent, and are worse for 2D methods than for central regions of 3D methods. This problem is illustrated in Fig. 1.

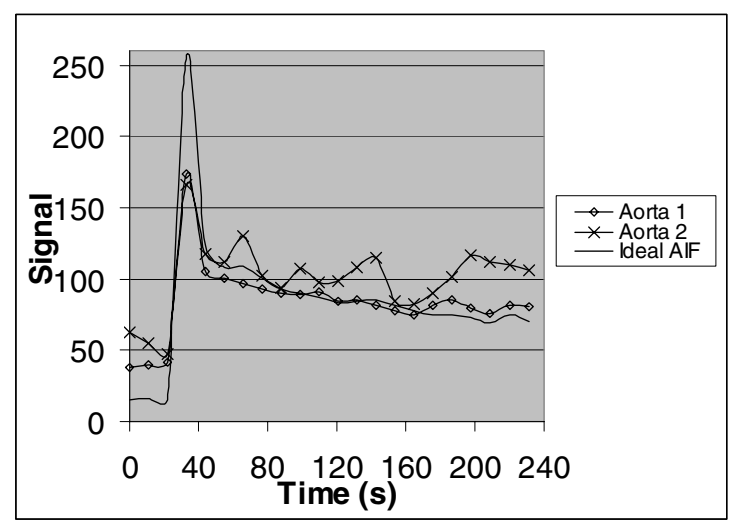

Fig. 1. Raw enhancement curves for two ROIs taken from the aorta of one human subject, with an automatically identified ideal AIF. Note that aorta 1 and aorta 2 show significantly different enhancement curves. Moreover, neither vessel shows the distinctive sharp peak and subsequent plateau characteristic of plasma enhancement following injection of a tracer bolus.

We have developed a method for the identification of an optimized AIF, described below, which is intended to eliminate this source of measurement variability and thereby increase the sensitivity to change of perfusion parameter measurements. This method is similar in aim to those presented previously by Rijpkema et al. [13] and Murase et al. [14]. Our method differs from the former it is completely automated, whereas the Rijpkema method requires some operator interaction, primarily to eliminate voxels consistent with veins rather than arteries. Our method is able to accomplish this without human interaction (see Fig. 2). Our method differs from the Murase technique in that it searches the entire volume for possible arterial voxels, whereas the Murase method begins with a user-defined arterial ROI, then divides that region into two or more sub-regions and averages the voxels in the optimal sub-region to generate its AIF. The AIF generated by our method for one human subject is shown in Fig. 1. Note that this curve shows a greater enhancement peak than that of either manually identified ROI, a smoother plateau, and a more characteristic shape. The primary purpose of this study was to assess the reproducibility of vascular perfusion 
parameter calculation using this automated AIF identification technique with respect to that which is achievable using manual AIF identification. As a secondary point, we have also provided an example of the use of this technique to assess response to treatment of naturally occurring canine mammary tumors with an anti-vascular agent.
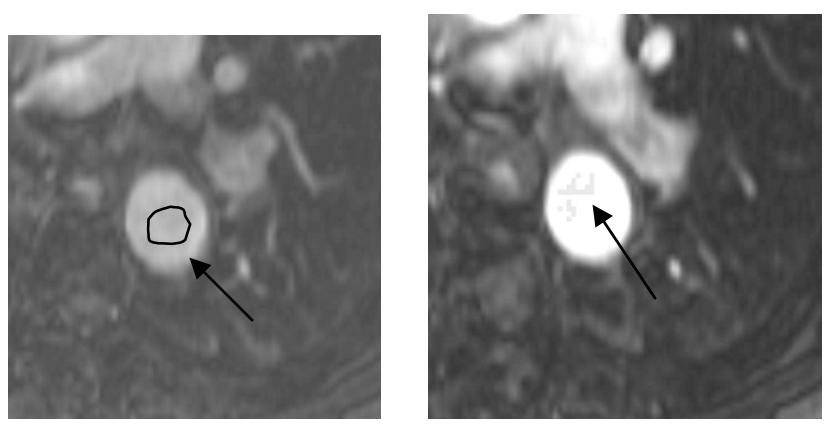

Fig. 2. (1) Manually drawn arterial ROI used to derive "Aorta 1" AIF shown in Fig. 1. (r) Light gray shaded voxels show the automatically identified voxel set used to derive "Ideal AIF" shown in Fig. 1.

\section{Measurement Techniques}

Tumor margins were identified in this study using a computer-aided boundary finding technique [15]. The resulting contour was then converted into a snake [16] which could be interactively corrected by the analyst if the initial result was sub-optimal.

After identifying the tumor margins, the analysts were required to identify a region of interest within an artery, preferably in close proximity to the tumor. This was done using manual tracing with a computer mouse. At this point, the identified arterial region was used for parameter calculation, as described below. In addition, the identified arterial region was used to initialize an automated search algorithm whose intent was to identify an optimized AIF for the data set under consideration. Each voxel in the data set was assigned a score based on time point of maximum enhancement, slope at maximum enhancement, peak value, and conformance to a gamma variate curve. This was accomplished by sorting all voxels in the data set based on the first three inter-related parameters. The highest scoring fifty voxels were then re-sorted based on the fourth parameter. The highest scoring twenty-five voxels in this sub-set were then assigned to the ideal AIF region of interest, and the automated AIF was generated by averaging the enhancement curves observed in these voxels (see Fig. 2).

After an arterial region of interest had been identified by either manual or automated means, enhancement curves were generated for both tumor and arterial plasma. These were designated $C_{t}(t)$ and $C_{p}(t)$, respectively. In the interests of noise reduction, both plasma and tumor data were fit to gamma-variate curves (17). The vascular bed was modeled as a linear system, such that:

$$
C_{t}(t)=C_{p}(t) * h(t)
$$


with impulse response $h(t)$ given by:

$$
h(t)=K^{\text {trans }} e^{-k_{e p} t}
$$

where $k_{e p}$ is the rate constant between the EES and blood plasma. Given $C_{t}(t)$ and $C_{p}(t), \quad K^{\text {trans }}$ and $k_{e p}$ were estimated using a gradient-descent energy minimization scheme in which local minima were avoided through the use of multiple instantiations with different initial parameter settings.

\section{Experimental Procedure}

The experiments involved in this study were intended to assess the reproducibility of perfusion measurements using manual and automated plasma identification, and to determine the percentage of measurement variability due to differences in tumor margin and plasma region of interest, respectively. Experimental data for the animal model were derived from three dogs with naturally occurring mammary tumors. These data were collected as part of an experiment testing an anti-angiogenic drug with vascular endothelial growth factor (VEGF) receptor tyrosine kinase inhibitory activity at a maximally tolerated dose in dogs. This drug was later discontinued from further development. Each animal was imaged three times over a period of 12 weeks. At each time point all animals were imaged in a single 48 hour period. Images for this study were acquired using a GE $1.5 \mathrm{~T}$ LX/CV scanner. Three slices through each tumor were acquired using a cardiac coil. Perfusion images used a GRE pulse sequence with a repetition time of $20 \mathrm{~ms}$, echo time of $1 \mathrm{~ms}$, and a flip angle of 40 degrees. Imaging time for each image set was seven seconds, with a two second scanner delay, yielding temporal resolution for the data set of nine seconds. The reconstruction matrix was 256x192, FOV was $140 \mathrm{~mm}$, and slice thickness was $4 \mathrm{~mm}$.

Experimental data from humans were taken from a subset of images collected as part of a Phase I clinical trial. Twelve subjects with tumors of the lungs, liver, head or neck were imaged twice, at baseline (within 3 days before day 1 treatment with a VEGF/PDGFR/c-Kit tyrosine kinase inhibitor) and on day 2 (cycle 1) after the morning dose, at the estimated $\mathrm{C}_{\max }$. Images for this study were acquired using a $\mathrm{GE}$ 1.5T Signa LX scanner. Ten slices through each tumor were acquired using appropriate coils with respect to the area being imaged. Dynamic contrast enhanced images used a 3DFSPGR pulse sequence with a repetition time set by the scanner of $6-7 \mathrm{~ms}$, echo time of min full (the minimum TE at which a full echo can be acquired - in this case $2 \mathrm{~ms}$ ), and a flip angle of 25 degrees. Imaging time for the dynamic contrast enhanced scan consisted of 11 breath-holds at approximately 30 seconds per breath-hold, with two phases being acquired per breath-hold. The reconstruction matrix was $256 \times 128$, FOV was kept to the smallest allowable so that no wrap in the images was present, and slice thickness was $5 \mathrm{~mm}$.

Because a primary aim of this study was the assessment of inter-operator variability, four analysts were trained in the use of the analysis software. Two analysts were experienced MR technicians, and two were radiologists with extensive oncology experience. All analysts were trained in the appearance of canine mammary 
tumors and the selection of appropriate plasma regions using images from animals not included in this study. Each analyst was then asked to identify and delineate both tumor and plasma in each of the nine included canine data sets as well as each of the 24 human subject data sets. When identifying arterial plasma, the analysts had the option to view the enhancement curve for the manually selected region at any time, and to erase, modify or replace the currently selected region. In this way each analyst was able to manually select a reasonably optimized arterial plasma region.

Once all regions of interest were delineated, $K^{\text {trans }}$ values were calculated first using the regions of interest as identified by the analyst, and then using the analyst's tumor identification with the automatically identified plasma enhancement curve. By comparing the variance seen between analysts using manually identified AIF with that seen between analysts using the automatically identified AIF, which was identical across analysts, it was possible to isolate variability related to AIF selection from that related to differences in tumor margin identification.

\section{Results and Discussion}

Coefficients of variability in measurement of $K^{\text {trans }}$ among the four analysts, defined as measurement standard deviation divided by measurement mean, were calculated separately for manual and automatic AIF identification, and for each of the nine canine and 24 human cases examined. For the nine canine manual AIF identifications, coefficients of variability ranged from $3.1 \%$ to $39.2 \%$, with a root mean square average of $23.7 \%$ and a median value of $21.5 \%$. For the nine canine automated AIF identifications, coefficients of variability ranged from $3.1 \%$ to $11.8 \%$, with a root mean square average of $7.0 \%$ and a median value of $6.2 \%$. For the 24 human manual AIF identifications, coefficients of variability ranged from $1 \%$ to $32 \%$, with a root mean square average of $14.1 \%$ and a median of $11 \%$. For the 24 human automated AIF identifications, coefficients of variability ranged from $1 \%$ to $24 \%$, with a root mean square average of $11.2 \%$ and a median of $6 \%$. It should be noted that in the human data there was a single case where the analysts disagreed significantly over the correct tumor boundaries. This case was the source of the high maximum variation in both the manual and automated AIF results.

Bearing in mind that the same tumor margins were used for both the automated and manual AIF calculations, in the canine experiment it can be generally surmised that approximately two thirds of the variability seen in the manual measurements was a result of differing AIF identifications, with the remaining one third attributable to differing tumor margin identifications. In the human experiments the median variability due to tumor margin identification was similar - approximately $6 \%$-- while the variability due to AIF identification was significantly lower. This is reasonable, as the smaller vessels and higher blood velocity present in the canines makes manual identification of an uncorrupted arterial region much more difficult than in the human case. Additionally, the 2D imaging method used in the canine study is more sensitive to in-flow effects than the 3D method used in the human study.

An examination of scatter-plots of $K^{\text {trans }}$ measurements using manual vs. automatic AIF identification for both canine and human data (see Fig. 3) shows that the correlation between the two measures is reasonable given the high variability of 
the manual measurements. It also shows a slope of 0.58 for the canine data and 0.85 for the human data, indicating that on average the manual measurement gives a somewhat higher estimation of $K^{\text {trans }}$ than the automatic measurement, and that this effect is more pronounced in the canine data than in the human data. This is as expected, since the general effect of flow artifacts will be to reduce the apparent plasma enhancement, thereby exaggerating the proportion of tracer apparently passing into the EES.
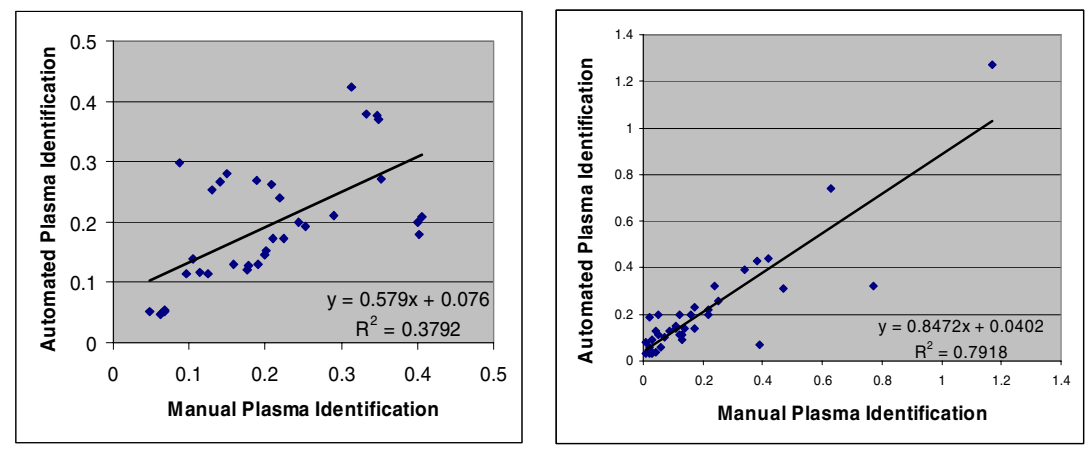

Fig. 3. Scatterplots of $K^{\text {trans }}$ values using manual and automatic plasma identification for canine (l) and human (r) data sets. Note that in both cases the trend line slope is less than 1, due to the fact that manual values are generally over-estimated due to poor plasma identification. This effect is less pronounced in the human data, and the correlation between manual and automated measurements is higher due to the improved manual reproducibility in the human data.

An examination of the trend over time in $K^{\text {trans }}$ for canine subject 1 (see Fig. 4) using manual and automated AIF identification highlights the value of the reduced measurement variability afforded by the automated process. Although both trend lines indicate that $K^{\text {trans }}$ for this tumor is declining over time, higher variability makes that assumption statistically insupportable at time two for manual AIF identification, and marginally supportable after time three. Using automated AIF identification, however, this subject may be confidently classified as declining after time 2 .

\section{Conclusions}

The two most commonly used methods for AIF definition in current clinical and experimental practice are manual AIF identification and use of a theoretical or modelbased AIF. The results of this study indicate that increased accuracy and sensitivity to change could be achieved by making use of an automated method for AIF identification such as the one described here. Use of such a method allows AIF identification to be both consistent and repeatable among operators, and also patient and time-point specific. Variability due to partial volume effects and artifacts is reduced with respect to that of manual AIF identification, while variability due to 
differences in injection and cardiac output is reduced with respect to that seen with the use of a model AIF.

In considering the differences between human and canine results given here, it should be noted again that the difficulty of identifying a suitable AIF is typically greater in smaller animals such as the canines used in this study than in humans. This is due to small animals' higher blood velocity, which exaggerates flow artifacts in the arteries, as well as to the lower signal to noise ratio that is achievable when imaging smaller anatomy.
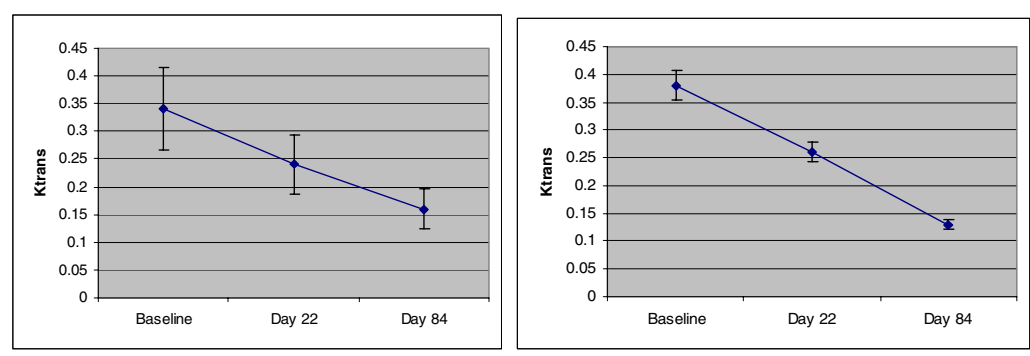

Fig. 4. Trend line using manual (l) and automated (r) plasma identification for canine subject 1. Note that the subject cannot be confidently classified as declining until time 3 using manual plasma identification, while the reduced variability of the automated measurements allows a confident classification after time 2 .

\section{References}

1. Orel SG, Differentiating benign from malignant enhancing lesions identified at MR imaging of the breast: are time-signal intensity curves an accurate predictor? Radiology 1999; 211:5- 7 .

2. Taylor J, Tofts P, Port R, Evelhoch J, Knopp M, Reddick W, Runge V, Mayr N. MR imaging of tumor microcirculation: Promise for the new millennium. J Magn Reson Imag 1999; 10:903 - 907.

3. Jackson A, Haroon H, Zhu X, Li K, Thacker N, Jayson G. Breath-hold perfusion and permeability mapping of hepatic malignancies using magnetic resonance imaging and a first-pass leakage profile model. NMR Biomed 2002; 15:164 - 173.

4. Tofts P. Modeling tracer kinetics in dynamic Gd-DTPA MR Imaging. J Magn Reson Imag 1997; 7:91 - 101.

5. Evelhoch J. Key factors in the acquisition of contrast kinetic data for oncology. J Magn Reson Imag 1999; 10:254 - 259.

6. Murase K. Efficient method for calculating kinetic parameters using T1-weighted dynamic contrast-enhanced magnetic resonance imaging. Magn Reson Med 2004; 51:858 - 862.

7. Tofts P, Brix G, Buckley D, Evelhoch J, Henderson E, Knopp M, Larsson H, Lee T, Mayr N, Parker G, Port R, Taylor J, Weisskoff R. Estimating kinetic parameters from dynamic contrast enhanced T1 weighted MRI of a diffusible tracer: standardized quantities and symbols. J Magn Reson Imag 1999; 10:223 - 232.

8. Weinmann HJ, Laniado M, Mutzel W. Pharmacokinetics of Gd-DTPA/dimeglumine after intravenous injection into healthy volunteers. Phisiol Chem Phys Med NMR 1984; $16: 167-172$. 
9. Tofts P, Kermode A. Measurement of the blood-brain barrier permeability and leakage space using dynamic MR imaging. 1. Fundamental Concepts. Magn Reson Med 1991; $17: 357-367$.

10. Padhani A, Hayes C, Landau S, Leach M. Reproducibility of quantitative dynamic MRI of normal human tissues. NMR Biomed 2002; 15:143 - 153.

11. Galbraith S, Lodge M, Taylor N, Rustin G, Bentzen S, Stirling J, Padhani A. Reproducibility of dynamic contrast-enhanced MRI in human muscle and tumours: comparison of quantitative and semi-quantitative analysis. NMR Biomed 2002; 15:132 - 142 .

12. Vonken E, Osch M, Bakker C, Viergever M. Measurement of cerebral perfusion with dual-echo multi-slice quantitative dynamic susceptibility contrast MRI. J Magn Reson Imag 1999; 10:109 - 117.

13. Rijpkema M, Kaanders J, Joosten F, van der Kogel A, Heerschap A. Method for quantitative mapping of dynamic MRI contrast agent enhancement in human tumors. J Magn Reson Imag 2001; 14:457 - 463.

14. Murase K, Kikuchi K, Miki H, Shimizu T, Ikezoe J. Determination of arterial input function using fuzzy clustering for quantification of cerebral blood flow with dynamic susceptibility contrast-enhanced MR imaging. J Magn Reson Imag 2001; 13:797 - 806.

15. Ashton E, Takahashi C, Berg M, Goodman A, Totterman S, Ekholm S. Accuracy and reproducibility of manual and semiautomated quantification of MS lesions by MRI. J Magn Reson Imag 2003; 17:300 - 308.

16. Kass M, Witkin A, Terzopolous D. Snakes: active contour models. Int J Comput Vision $1988 ; 321-331$.

17. Moran G, Prato F. Modeling tissue contrast agent concentration: a solution to the tissue homogeneity model using a simulated arterial input function. Magn Reson Med 2001; 45: 42 - 45. 